\title{
High resolution magnetic force microscopy using focused ion beam modified tips
}

\author{
G. N. Phillips, ${ }^{\text {a) }}$ M. Siekman, L. Abelmann, and J. C. Lodder \\ Systems and Materials for Information Storage Group, MESA ${ }^{+}$Research Institute, University of Twente, \\ P.O. Box 217, 7500AE Enschede, The Netherlands
}

(Received 28 January 2002; accepted for publication 4 June 2002)

\begin{abstract}
Atomic force microscope tips coated by the thermal evaporation of a magnetic $30 \mathrm{~nm}$ thick Co film have been modified by focused ion beam milling with $\mathrm{Ga}^{+}$ions to produce tips suitable for magnetic force microscopy. Such tips possess a planar magnetic element with high magnetic shape anisotropy, an extremely high aspect ratio of greater than 30:1, and an end radius of less than $25 \mathrm{~nm}$. These tips have been used in a commercial atomic force microscope under ambient conditions to obtain $30 \mathrm{~nm}$ resolution magnetic images of an established $\mathrm{CoNi} / \mathrm{Pt}$ multilayer reference sample. (C) 2002 American Institute of Physics. [DOI: 10.1063/1.1497434]
\end{abstract}

Magnetic force microscopy ${ }^{1}$ (MFM) has become one of the most widely used techniques for imaging surface magnetic stay field distributions. This is due to the minimal sample preparation and the relative ease with which high quality sub-100-nm resolution images may be obtained under normal laboratory conditions. With the now widely available commercial microscopes and magnetic tips, ${ }^{2}$ images with 50 $\mathrm{nm}$ resolution are quite routine; however, obtaining images with higher resolution is more demanding experimentally. ${ }^{3-7}$

In this letter, we present sub-30-nm resolution images obtained under ambient conditions with a Digital Instruments DI3100 magnetic force microscope and Nanosensors GmbH Pointprobe noncontact tapping mode silicon tips coated on one face with a Co thin film which is then modified by focused ion beam (FIB) milling. The images are of magnetic features in a well-known specially prepared MFM reference sample possessing perpendicular magnetic anisotropy. ${ }^{7,8}$

An ideal MFM tip should possess a small physical size along with well-defined stable magnetic properties. ${ }^{9}$ Sufficient magnetic material must be present to allow the interaction between the tip and the sample stray field to be detected with an adequate signal to noise ratio. The ideal MFM tip shape is a thin bar with small cross sectional area and high aspect ratio. ${ }^{9}$ Such a tip forms a magnetic dipole, as magnetic charge is concentrated at the two ends. If sufficiently long, the tip will behave as a monopole as the far end of the tip will experience a negligible stray magnetic field from the sample. A small cross sectional area defines the minimum detectable magnetic feature size, while a high aspect ratio ensures a stable magnetization and increases the sensitivity of the tip to long wavelength features. Electron beam induced deposited (EBID) tips have approached this ideal. ${ }^{2-5}$

FIB milling has been used previously to prepare MFM tips. Kikukawa et al. ${ }^{10}$ defined the physical structure of the tip by FIB and then deposited a magnetic thin film, as with EBID tips. Khizroev et al. ${ }^{11}$ modified tips coated with a 10 $\mathrm{nm}$ thick film of high magnetic anisotropy $\mathrm{CoCrPt}$ resulting

\footnotetext{
${ }^{a)}$ Current address: Philips Research Laboratories, Prof. Holstlaan 4 (WY22), 5656AA Eindhoven, The Netherlands; electronic mail: gavin.phillips@phillips.com
}

in extremely low aspect ratio $(\mathrm{AR}=0.166: 1)$ tips approximating to point magnetic dipoles. These tips were developed for the quantification of magnetic fields from write heads, and were not optimized for image resolution. Recently, Folks et al. ${ }^{12}$ obtained images of data tracks possessing $50 \mathrm{~nm}$ bit lengths with tips modified by FIB in such a way as to be sensitive to in-plane components of the sample stray magnetic field, however, it was reported to be difficult to ascertain the ultimate resolution of these tips. ${ }^{12}$

In this work, etched silicon Pointprobe noncontact tapping mode cantilevers produced by Nanosensors $\mathrm{GmbH}$ had a 30-nm-thick film of cobalt evaporated onto one face of the pyramidal tip. The Co film was then modified by FIB milling in an FEI200 workstation with $\mathrm{Ga}^{+}$ions possessing an energy of $30 \mathrm{kV}$. The majority of the Co thin film was etched away to leave thin bars with extremely high aspect ratios (AR>30:1), as shown in Fig. 1. For a similar thin Co film deposited and etched under the same conditions as the tip coating, the etch rate at a beam current of $1 \mathrm{pA}$ was 0.41
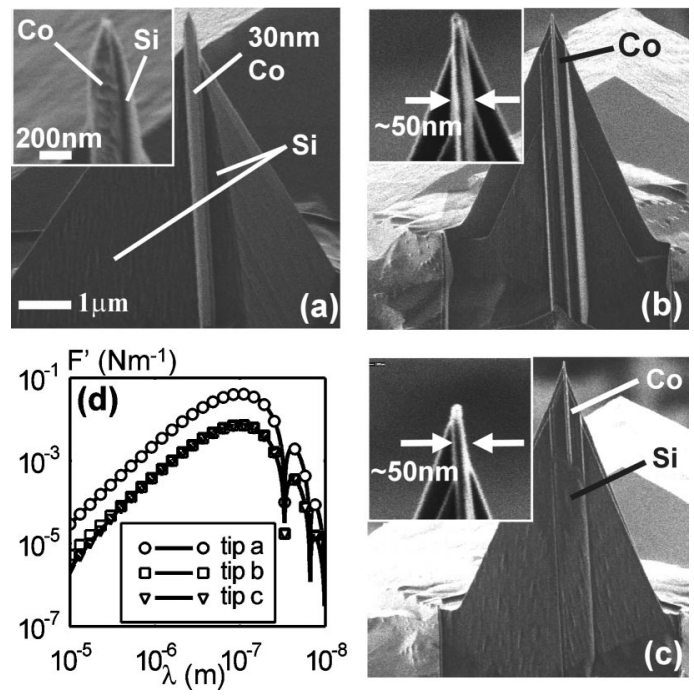

FIG. 1. (a)-(c): Secondary electron images of FIB modified MFM tips showing the high aspect ratio $30 \mathrm{~nm}$ thick Co elements after etching. The insets show the tip apexes at higher magnification; (d) modeled spatial response of tips a to $\mathrm{c}$ at a $15 \mathrm{~nm}$ scan height. 


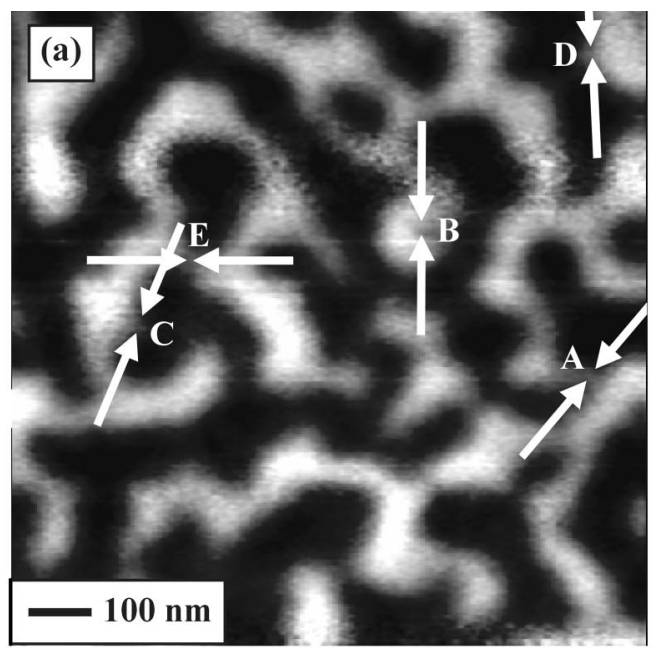

Frequency shift $(\mathrm{Hz})$

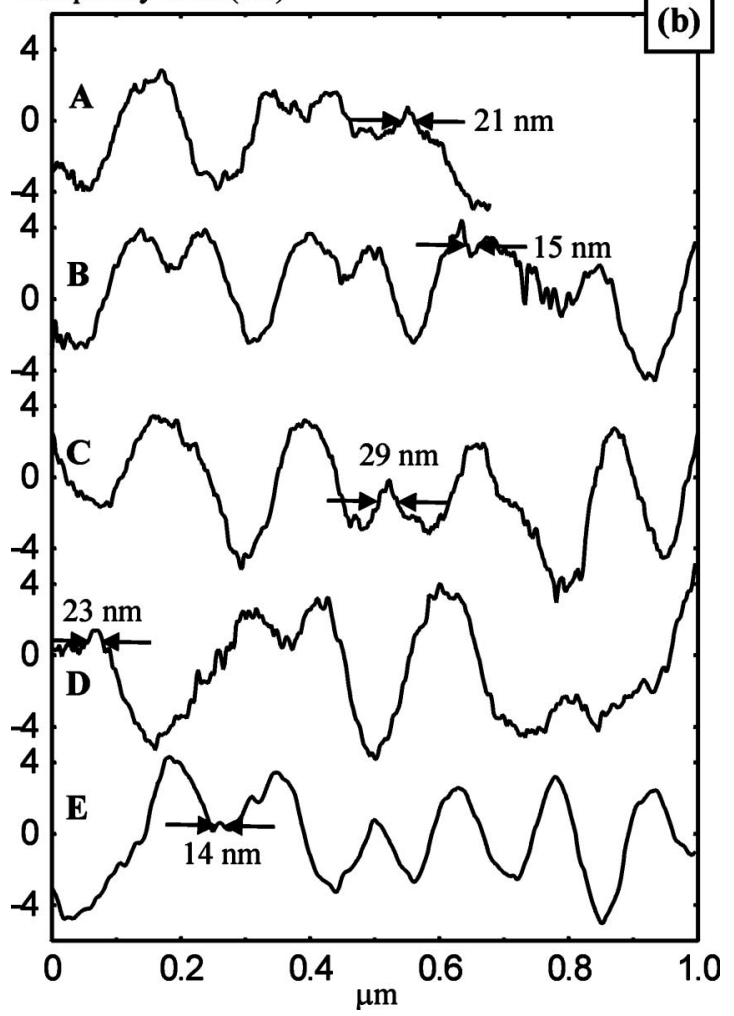

FIG. 2. (a) MFM image of domains at the periphery of a bit written into a $\mathrm{CoNi} / \mathrm{Pt}$ multilayer magneto-optic thin film. This image was obtained with tip a. The arrows indicate the position of features (A) $-(\mathrm{E})$ shown in (b) that possess a FWHM of less than $30 \mathrm{~nm}$. Scan line E exhibits the least noise as it lies directly along the $x$ axis of the image.

$\pm 0.01 \mu \mathrm{m}^{3} \mathrm{nC}^{-1}\left(\equiv 5.7 \pm 0.1 \mathrm{nms}^{-1}\right)$. It is expected that the etch rate of the tip coating was higher than this figure as etch rate increases with the angle of incidence of the $\mathrm{Ga}^{+}$ ions relative to the surface normal. ${ }^{13}$ Figure 1(a) reveals that the bar on this tip (a) tapers down from a width of $\sim 278 \mathrm{~nm}$ to an end radius of less than $25 \mathrm{~nm}$. Figures 1(b) and 1(c) illustrate that it is possible to FIB magnetic elements with a uniform width of $50 \mathrm{~nm}$; tips (b) and (c) have elements 8.34 $\mu \mathrm{m}$ long $(\mathrm{AR}=167: 1)$ and $1 \mu \mathrm{m}$ long $(\mathrm{AR}=20: 1)$, respectively. Figure 1(d) shows the calculated frequency response for ideal bar shaped tips modeled according to Refs. 7 and 9, and possessing the same physical characteristics as tips a-c. Generally, due to a magnetic layer thickness of $30 \mathrm{~nm}$, the tips exhibit minima in their calculated response at 30 and 15
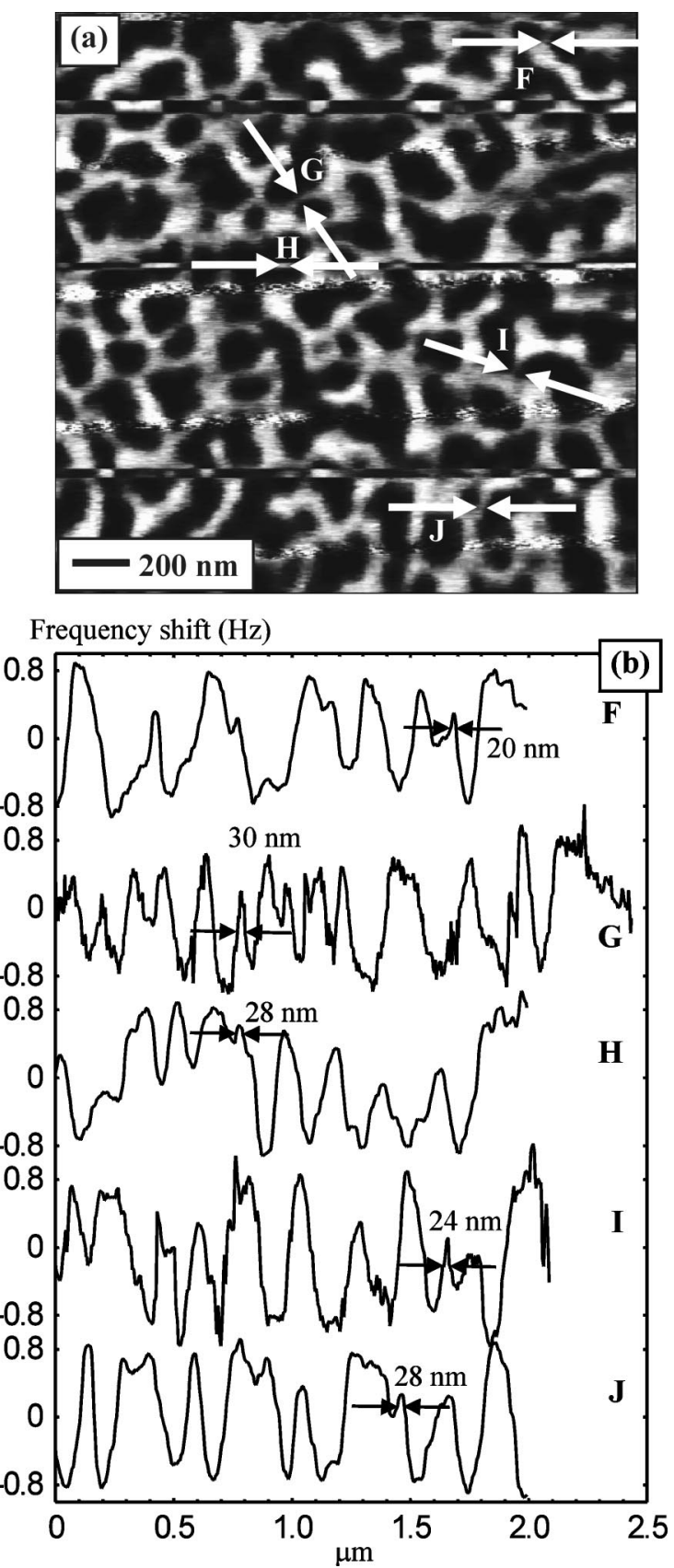

FIG. 3. (a) MFM image of domains at the periphery of a bit written into a $\mathrm{CoNi} / \mathrm{Pt}$ multilayer magneto-optic thin film. This image was obtained with tip c. The arrows indicate the position of features $(F)-(J)$ shown in $(b)$ that possess a FWHM of less than $30 \mathrm{~nm}$. Scan line $\mathrm{H}$ is through a region where the magnetization of the tip has been reversed.

$\mathrm{nm}$. This is analogous to the "gap zero" for magnetic recording heads. Additionally, due to a larger magnetic volume, the magnitude of the calculated response for tip a is $\sim 5$ times that of tips $b$ and $c$.

MFM images were obtained at room temperature, in air, using tips a and c mounted in a Digital Instruments D3100 SPM operated in the so-called tapping/lift mode. All images are of domains located at the periphery of a bit written in a 20 bilayer $35 \mathrm{~nm}$ thick $\mathrm{Co}_{50} \mathrm{Ni}_{50} / \mathrm{Pt}(0.55 \mathrm{~nm} / 1.2 \mathrm{~nm})$ magneto-optic thin film possessing perpendicular anisotropy and $H_{c}=1.256 \mathrm{kOe}\left(\sim 100 \mathrm{kAm}^{-1}\right)$. The suitability of this sample as a reference for assessing MFM resolution has been discussed elsewhere. ${ }^{7}$ Prior to imaging, the tip magnetization was defined with the stray field from a permanent magnet. to AIP license or copyright, see http://ojps.aip.org/aplo/aplcr.jsp 
Figures 2(a) and 3(a) show MFM images taken while monitoring the shift in frequency due to the interaction between tip and sample stray field. A minimum tip to sample spacing of at least $15 \mathrm{~nm}$ was used. Due to the careful operation of the microscope, topographic data are not visually apparent in the MFM image. The features in both images are typical of those observed for magnetic domains in magnetooptic thin films possessing high perpendicular magnetic anisotropy and relatively strong domain wall pinning. ${ }^{14}$ It is thought that the bands of noisy scan lines observed in both images may be due to optical interference between light reflected from the sample and backside of the cantilever. Additionally, scan lines where the magnetization of tip c has been reversed can be observed in Fig. 3(a). ${ }^{15}$

The arrows in Figs. 2(a) and 3(a) indicate the positions of a number of sub-30-nm features (A)-(J). Figures 2(b) and 3(b) illustrate that the size of these features was quantified by measuring the full width half maximum (FWHM) of the corresponding peaks and troughs in line traces through the image data. The measurement of image artifacts such as noise spikes was avoided by careful reference to the image. Line traces $\mathrm{A}-\mathrm{D}, \mathrm{G}$, and I are somewhat noisy due to the $1 / f$ component of the noise revealed by positioning line traces across scan lines. Features with a FWHM of less than $30 \mathrm{~nm}$ are easily identified, with features as small as $14 \mathrm{~nm}$ being observed in scan lines B and E. The line traces also reveal that the maximum frequency shift observed for tip a is 5 times larger than that for tip c, in agreement with the calculated data of Fig. 1(d).

Distinguishing magnetic features from noise and the measurement of their size is open to a large degree of subjectivity. Some interscan line correlation should exist for magnetic features while being nonexistent for noise spikes. The examination of both images revealed that there was good correlation between adjacent scan lines for features A-J, suggesting that they are not noise spikes. Furthermore, both Gaussian filtering and scan-line smoothing have been used separately to gauge the effect of removing any high frequency noise spikes that may exist. In both cases the same sub-30-nm features were clearly identified. Additionally, the approach used in Ref. 7 has been used to determine a mean size of $29.3 \mathrm{~nm}$ with a standard deviation of $2.2 \mathrm{~nm}$ for ten features in Fig. 2(a).

Close examination of simultaneously acquired atomic force microscopy (AFM) data reveal that the $14 \mathrm{~nm}$ features in scan lines B and E occur at the same position as similarly sized surface topographic features. This may be due to the influence of capillary or van der Waals forces between tip and sample however at a tip to sample spacing of $15 \mathrm{~nm}$ such forces are very small. ${ }^{9}$ Electrostatic interactions between tip and sample may exist, although this is thought to be unlikely due to the similarity of the magnetic materials. The AFM image of the sample surface revealed a granular texture typi- cal of a multilayer film possessing a columnar structure. Similar films have been shown to grow with a columnar structure under certain conditions. ${ }^{16,17}$ Domain wall pinning at the column boundaries in such films results in a physical mechanism by which domain sizes are controlled and correlate to surface texture. Thus, for such samples it is expected that magnetic features may indeed be observed at the same position as those on the sample surface.

This work has shown that MFM images with a resolution of $30 \mathrm{~nm}$ may be obtained under ambient conditions by the use of tips suitably modified by FIB milling. It is expected that such tips will be of great interest to the magnetic data storage community where hard disk demonstrations have been made with bit lengths of $50 \mathrm{~nm}$ or less. ${ }^{12,18}$ Future continuous media, or patterned magnetic elements, suitable for MRAM applications, will exhibit ever-smaller magnetic feature sizes, hence the ability to image sub-50-nm magnetic regions will continue to be of great importance.

The financial support of the Dutch STW Research Foundation under Project No. TEL. 4562 is gratefully acknowledged. The research of L. Abelmann has been made possible by a fellowship of the Royal Netherlands Academy of Arts and Sciences.

${ }^{1}$ Y. Martin and H. K. Wickramsinghe, Appl. Phys. Lett. 50, 1455 (1987).

${ }^{2}$ K. Babcock, V. Elings, M. Dugas, and S. Loper, IEEE Trans. Magn. 30, 4503 (1994).

${ }^{3}$ P. B. Fischer, M. S. Wei, and S. Y. Chou, J. Vac. Sci. Technol. B 11, 2570 (1993).

${ }^{4}$ M. Ruhrig, S. Porthun, and J. C. Lodder, Rev. Sci. Instrum. 65, 3224 (1994).

${ }^{5}$ G. D. Skidmore and E. D. Dahlberg, Appl. Phys. Lett. 71, 3293 (1997).

${ }^{6}$ H. J. Hug, B. Stiefel, P. J. A. van Schendel, A. Moser, S. Martin, and H. J. Guntherodt, Rev. Sci. Instrum. 70, 3625 (1999).

${ }^{7}$ L. Abelmann, S. Porthun, M. A. M. Haast, J. C. Lodder, A. Moser, M. E. Best, P. J. A. van Schendel, B. Stiefel, H. J. Hug, G. P. Heyden, A. Farley, S. R. Hoon, T. Pfaffelhuber, R. Proksch, and K. Babcock, J. Magn. Magn. Mater. 190, 135 (1998).

${ }^{8}$ CAMST II reference sample.

${ }^{9}$ S. Porthun, L. Abelmann, and J. C. Lodder, J. Magn. Magn. Mater. 182, 238 (1998).

${ }^{10}$ A. Kikukawa, S. Hosaka, Y. Honda, and S. Tanaka, Appl. Phys. Lett. 61, 2607 (1992).

${ }^{11}$ S. K. Khizroev, W. Jayasekara, J. A. Bain, R. E. Jones, and M. H. Kryder, IEEE Trans. Magn. 34, 2030 (1998).

${ }^{12}$ L. Folks, M. E. Best, P. M. Rice, B. D. Terris, and D. Weller, Appl. Phys. Lett. 76, 909 (2000).

${ }^{13}$ P. E. Russell, T. J. Stark, D. P. Griffiths, J. R. Phillips, and K. F. Jarausch, J. Vac. Sci. Technol. B 16, 2494 (1998).

${ }^{14}$ G. N. Phillips, K. O'Grady, Q. Meng, and J. C. Lodder, IEEE Trans. Magn. 32, 4070 (1996).

${ }^{15}$ M. Ruhrig, S. Porthun, J. C. Lodder, S. McVitie, L. J. Heyderman, A. B. Johnstone, and J. N. Chapman, J. Appl. Phys. 79, 2913 (1996).

${ }^{16}$ W. B. Zeper, H. M. van Kesteren, B. A. J. Jacobs, J. H. M. Spruit, and P. F. Carcia, J. Appl. Phys. 70, 2264 (1991).

${ }^{17}$ Q. Meng, P. de Haan, W. P. van Drent, J. C. Lodder, and Th. J. A. Popma, IEEE Trans. Magn. 32, 4064 (1996).

${ }^{18}$ Fujitsu $106 \mathrm{Gbit} / \mathrm{in}^{2}$ demonstration, J. Hong et al., presentation BQ-12, TMRC conference (Aug. 2001) 